\title{
Carbon nanotube-induced preparation of vanadium oxide nanorods: Application as a catalyst for the partial oxidation of $\boldsymbol{n}$-butane
}

\author{
Xiao-Wei Chen ${ }^{\mathbf{a}}$, Zhenping Zhu ${ }^{\mathbf{1}, \mathbf{a}}$, Michael Hävecker ${ }^{\mathbf{a}}$, Dang Sheng Su*, a, and Robert Schlögl ${ }^{\mathbf{a}}$ \\ ${ }^{a}$ Department of Inorganic Chemistry (Member of the European Laboratory of Catalysis and Surface \\ Science, ELCASS), Fritz-Haber-Institute of MPG, Faradayweg 4-6, D-14195 Berlin, Germany \\ * Corresponding author. Tel.: +49 308413 5406; fax: +49 308413 4401; email: dangsheng@fhi-berlin.mpg.de \\ ${ }^{1}$ Current address: State Key Laboratory of Coal Conversion, Institute of Coal Chemistry, Chinese Academy of Sciences, 030001 Taiwan, China.
}

\begin{abstract}
A vanadium oxide-carbon nanotube composite was prepared by solution-based hydrolysis of $\mathrm{NH}_{4} \mathrm{VO}_{3}$ in the presence of carbon nanotubes. The carbon nanotubes induce the nucleation of the 1D vanadium oxide nanostructures, with the nuclei growing into long freestanding nanorods. The vanadium oxide nanorods with the lengths up to $20 \mu \mathrm{m}$ and the widths of 5-15 $\mathrm{nm}$ exhibit a well-ordered crystalline structure. Catalytic tests show that the composite with nanostructured vanadium oxide is active for the partial oxidation of $n$-butane to maleic anhydride at $300^{\circ} \mathrm{C}$.
\end{abstract}

Keywords: composites; electron microscopy; X-ray diffraction; catalytic properties

\section{Introduction}

Carbon nanotubes (CNTs) exhibit excellent thermal conductivity, high surface area, and high thermal and chemical stability. Furthermore, carbon is also active and selective for catalytic oxidation reactions [1]. Nanostructured vanadium oxide is of particular interest [2], [3], [4], [5], [6], [7], [8], [9] and [10] because vanadium oxides are widely applied to electrochemical devices [7] and [10] and partial oxidations or dehydrogenations of alkanes to olefins [11], [12], [13] and [14]. Most of these reactions are highly exothermic. The energy is released to the catalyst surface causing the catalytic activity or selectivity to decline since the thermal conductivity of the active catalysts and/or the normally used supports is very low. This problem is possibly solved by employing supports with high thermal conductivity, such as $\mathrm{CNTs}, \beta-\mathrm{SiC}, \mathrm{Si}_{3} \mathrm{~N}_{4}$, and $\mathrm{BN}$ [11] which can act to conduct the heat from the catalyst surface to the reactor walls.

A combination of vanadium oxides with CNTs is expected to produce a novel composite material exhibiting improved physical and chemical properties. In 1995, vanadium oxide-carbon nanotube composites were prepared by annealing a mixture of CNTs and $\mathrm{V}_{2} \mathrm{O}_{5}$ powder in air above the melting point of vanadium oxide [3]. Such nanocomposites can also be synthesized by coating the surface of nitric acid-treated CNTs with vanadic acid [5]. The CNTs in these nanocomposites act as geometric templates that can be removed to produce vanadium oxide nanorods. Mesoporous carbons (CMK) [15], [16], [17] and [18] have also been used as templates to prepare $\mathrm{Pd}$, nickel oxide, iron oxide, and $\mathrm{MnO}_{2}-\mathrm{CMK}$ nanocomposites [19], [20], [21], [22] and [23]. Recently, synthesis of vanadium oxide-carbon nanotube composite electrodes for use in secondary lithium batteries by sol-gel method has been reported [24] and [25]. The electrodes exhibited high specific capacity at high discharge rates due to the effective conducting network of nanotubes. Vanadium oxide-CNT composite could be a promising catalytic material for exothermic reactions. However, the catalytic properties of such composites have not been tested.

Vanadium oxide nanotubes have been obtained previously through sol-gel reaction followed by hydrothermal treatment starting from vanadium alkoxide precursors and primary amines or $\alpha, \omega-$ 
diamins [6]. In addition, vanadium oxide nanowires have also been synthesized by reverse micelle technique [4]. Most of these vanadium oxide nanotubes or nanorods synthesized through hydrothermal or sol-gel method using organic vanadium as precursors, which are air sensitive and expensive. In this work, a novel and simple method for the preparation of vanadium oxideCNT composite based on hydrolysis of $\mathrm{NH}_{4} \mathrm{VO}_{3}$ solution in the presence of CNTs is reported. The CNTs play a catalytic role in the nucleation of vanadium oxide to form vanadium oxide nanorods. The catalytic performance of the composites for partial oxidation of $n$-butane to maleic anhydride is tested in a specially designed microreactor which allows a very small amount of composite to be used. Maleic anhydride, a precursor to polyester resins, is produced by partial oxidation of $n$-butane over vanadium pyrophosphate (VPO) catalysts [26], [27] and [28]. Generally, $(\mathrm{VO})_{2} \mathrm{P}_{2} \mathrm{O}_{7}$ is considered the active phase in selective oxidation of $n$-butane to maleic anhydride in VPO [28]. The as-obtained vanadium oxide-CNT composites in this work contain nanostructured vanadium oxide and carbon nanotubes. It may open a new horizon for application of such vanadium oxide-CNT composite in catalytic processes.

\section{Experimental}

Saturated $\mathrm{NH}_{4} \mathrm{VO}_{3}$ aqueous solution $(100 \mathrm{ml})$ was refluxed for $24 \mathrm{~h}$ to obtain the orange color hydrolysis product. The hydrolysis product was washed with deionized water and dried at 110 ${ }^{\circ} \mathrm{C}$ overnight. Multi-walled CNTs with diameters of 10-50 nm were supplied by Applied Science Ltd. The raw CNTs were pretreated with $\mathrm{HNO}_{3}$ solution $(6 \mathrm{M})$ at refluxing temperature for $12 \mathrm{~h}$. This would be remove partially the impurities (cobalt and amorphous carbon particles) and modify oxygen-containing groups on the surface of the carbon nanotubes. The acid-treated CNTs were washed with deionized water and ethanol, and dried at $60{ }^{\circ} \mathrm{C}$ for $24 \mathrm{~h}$. Then $100 \mathrm{ml}$ of saturated $\mathrm{NH}_{4} \mathrm{VO}_{3}$ aqueous solution containing $100 \mathrm{mg}$ of nitric acid-treated CNTs was stirred and refluxed for $5 \mathrm{~h}$ (the weight ratio of $\mathrm{V}: \mathrm{C}$ is $2: 1$; the $\mathrm{V}_{2} \mathrm{O}_{5}$ loading in the composite is around 78.4 wt.\%). After refluxing, the suspension was filtrated, washed with deionized water and ethanol, and dried at $110^{\circ} \mathrm{C}$ overnight.

A Philips TEM/STEM CM $200 \mathrm{LaB}_{6}$ transmission electron microscope was used to study the morphology and microstructure of the samples. The acceleration voltage was set $200 \mathrm{kV}$. The element maps of carbon, vanadium and oxygen were obtained by EDS in STEM mode in order to study the elemental distribution of the sample. Electron energy loss spectra of the sample were acquired in the energy loss interval $250-600 \mathrm{eV}$. XRD patterns of the as-prepared sample were recorded using a STOE diffractometer $(\mathrm{Cu} \mathrm{K} \alpha$ radiation).

The microreactor used for the catalytic tests of the material was described in detail elsewhere [29]. The catalyst was mounted using a sapphire sample holder that was heated by a NIR laser from the back. The sample was dispersed in ethanol. One drop of the suspension was released into a ring at the center of a stainless steel support and dried in air. The ring has an inner diameter of $3 \mathrm{~mm}$. The stainless steel support was mounted onto the sapphire sample holder. A thermocouple was spot-welded onto this ring for accurate temperature recording. A mixture of $n$ butane (1 vol.\%), oxygen (16 vol.\%) and nitrogen (83 vol.\%) was fed into the reactor by calibrated mass flow controllers at a total flow rate of $22 \mathrm{~cm}^{3} / \mathrm{min}$ at a total pressure of 1 bar. The reactor exhaust was introduced into a differentially pumped electron impact mass spectrometer (PRISMA, PFEIFFER) and a proton transfer reaction-mass spectrometer (PTR-MS, IONICON ANALYTIC). The PTR-MS is based on the principle of chemical ionisation using $\mathrm{H}_{3} \mathrm{O}^{+}$as the proton donator combined with the swarm technique of the flow tube type. The proton-transfer reactions are usually nondissociative [30] and [31]. This results in an almost fragmentation-free ionisation that makes this method suitable for a qualitative and quantitative analysis of volatile hydrocarbons at the ppb level. The as-synthesized vanadium oxide-carbon nanotube composite and commercial $\mathrm{V}_{2} \mathrm{O}_{5}$ were heated from room temperature to 200, 300 and $400{ }^{\circ} \mathrm{C}$ in reaction atmosphere, sequentially. 
Materials Research Bulletin 42, 354-361 (2007)
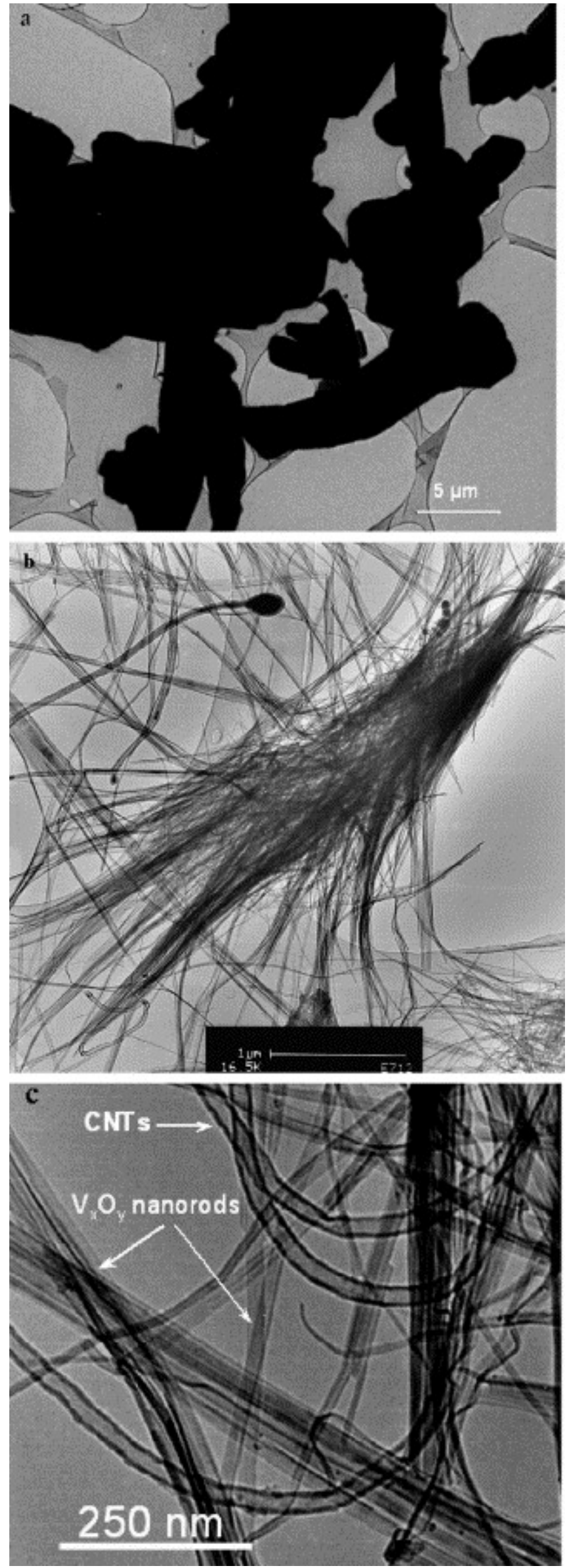

Fig. 1. TEM image of the products obtained from $\mathrm{NH}_{4} \mathrm{VO}_{3}$ hydrolysis in (a) the absence and (b and c) the presence of CNTs. Scale bars: (a) $5 \mu \mathrm{m}$, (b) $1 \mu \mathrm{m}$ and (c) $250 \mathrm{~nm}$. 


\section{Results and discussion}

The hydrolysis of saturated $\mathrm{NH}_{4} \mathrm{VO}_{3}$ solution alone could effectively produce $\mathrm{V}_{x} \mathrm{O}_{y}$ upon reflux but led to heavy aggregation and the formation of irregular micrometer particles (Fig. 1a). Control of size and shape of the particles is difficult. The specific BET surface area of the hydrolysis product of $\mathrm{NH}_{4} \mathrm{VO}_{3}$ is $16.8 \mathrm{~m}^{2} \mathrm{~g}^{-1}$. However, when the surface-modified CNTs (the specific BET surface area of the modified CNTs is $40.0 \mathrm{~m}^{2} \mathrm{~g}^{-1}$ ) were introduced into the aqueous $\mathrm{NH}_{4} \mathrm{VO}_{3}$ solution, $\mathrm{V}_{x} \mathrm{O}_{y}$ nanorods grew in the form of bundles (Fig. $1 \mathrm{~b}$ and c). The specific BET surface area of $\mathrm{V}_{x} \mathrm{O}_{y}-\mathrm{CNT}$ composite is $76.0 \mathrm{~m}^{2} \mathrm{~g}^{-1}$. The lengths of the $\mathrm{V}_{x} \mathrm{O}_{y}$ nanorods are up to $20 \mu \mathrm{m}$ and the average widths are 5-15 nm. Fig. 1c shows the separated $\mathrm{V}_{x} \mathrm{O}_{y}$ nanorods and CNTs. The vanadium oxide nanorods are 5-15 $\mathrm{nm}$ in diameters and therefore can be easily distinguished from the CNTs which are 10-50 $\mathrm{nm}$ in diameter. The energy of interaction between CNTs and $\mathrm{V}_{x} \mathrm{O}_{y}$ complexes would overcome the thermal activation barrier for aggregation of $\mathrm{V}_{x} \mathrm{O}_{y}$ and lead to rapid solidification along a layer on the surface of the CNTs. The heat of crystallization will assist the hydrolytic cleavage of the $\mathrm{V}_{x} \mathrm{O}_{y}$-CNT bonding generating pure $\mathrm{V}_{x} \mathrm{O}_{y}$ solid and allow for regeneration of free CNT surface. CNT-assisted productions of $1 \mathrm{D} \mathrm{V}_{2} \mathrm{O}_{5}$ nanostructures using molten $\mathrm{V}_{2} \mathrm{O}_{5}$ or $\mathrm{HVO}_{3}$ gel on tube surfaces and/or filled into internal void space were previously reported by other authors. In these cases, the CNTs act as geometric templates [3] and [5]. In the present approach, however, CNTs serve as inducers for the nucleation of the $\mathrm{V}_{x} \mathrm{O}_{y}$ nanostructures by providing curved surfaces. As seen in Fig. $2 \mathrm{~b}, \mathrm{~V}_{x} \mathrm{O}_{y}$ sprouts first grew along the axis of carbon nanotubes, and then peeled away from the carbon nanotubes due to weak interaction between them. Fig. $2 \mathrm{c}$ shows that the initial sprouts grew into long nanorods after peeling away from the carbon nanotubes. For this reason, only the separated $\mathrm{V}_{x} \mathrm{O}_{y}$ nanorods can be observed by transmission electron microscope (TEM). These observations suggest that CNTs do not act as templates in the present process but seem to serve as catalysts by providing their curved surfaces to induce the nucleation of the $\mathrm{V}_{x} \mathrm{O}_{y}$ nanostructures.

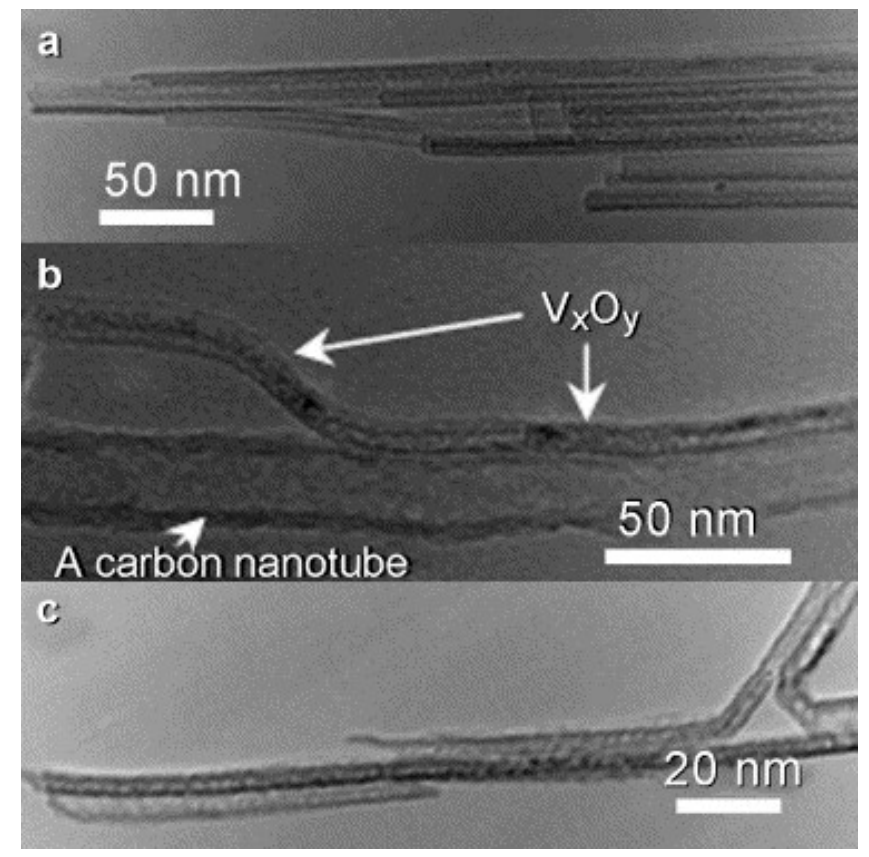

Fig. 2. (a) A bundle of $\mathrm{V}_{x} \mathrm{O}_{y}$ nanorods; (b) nucleation and peeling of a nanonod on (from) the surface of the carbon nanotube; (c) new 1D nuclei formed on the surface of a grown $\mathrm{V}_{x} \mathrm{O}_{y}$ nanorod.

Fig. 3(a) shows $\mathrm{XRD}$ patterns of the products from $\mathrm{NH}_{4} \mathrm{VO}_{3}$ hydrolysis in the absence and presence of CNTs. The XRD results indicate that the mixed composition of the products obtained from $\mathrm{NH}_{4} \mathrm{VO}_{3}$ hydrolysis match best with the patterns of $\mathrm{V}_{2} \mathrm{O}_{5} \cdot \mathrm{H}_{2} \mathrm{O}$ and $6 \mathrm{VO}_{2} \cdot 5 \mathrm{H}_{2} \mathrm{O}$ 
(JCPDS card number are 11-673 and 31-1441). Electron energy loss spectra were recorded in order to probe the local electronic structure of vanadium and oxygen sites (Fig. 3(b)). The shape of the vanadium and oxygen edges is strongly related to the oxidation state of the vanadium and to the distortion of coordination polyhedra. From the observed intensity ratio between the $\mathrm{VL}_{2}$ and $\mathrm{VL}_{3}$ peaks (the white lines) and from the position of the white lines it follows that the nanorods contain both $\mathrm{V}^{5+}$ and $\mathrm{V}^{4+}[32]$. This is in agreement with the XRD results.
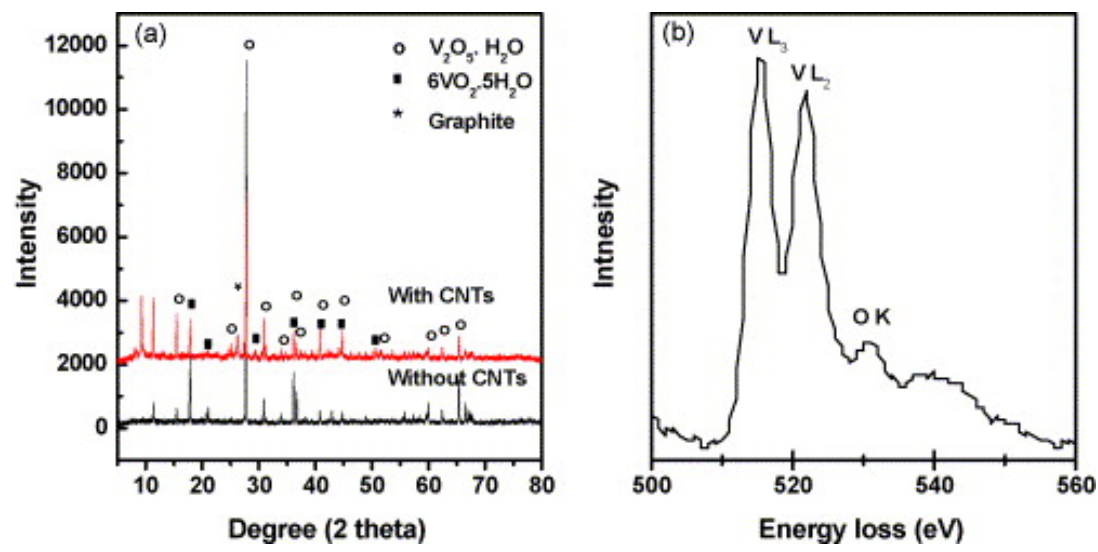

Fig. 3. (a) XRD patterns of the products of $\mathrm{NH}_{4} \mathrm{VO}_{3}$ hydrolysis in the absence and presence of CNTs; (b) EELS spectra of vanadium oxide nanorods for the V L-edge and the O K-edge

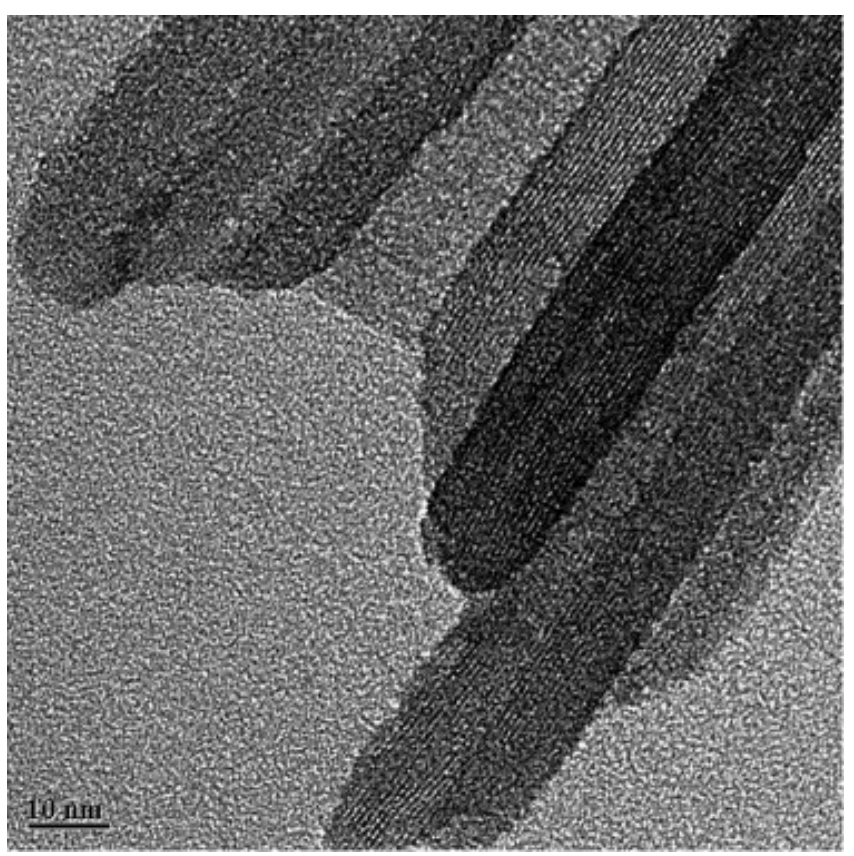

Fig. 4.High-resolution transmission electron microscopy of vanadium oxide nanorods.

The high-resolution transmission electron microscopy image (Fig. 4) shows that layer-like $\mathrm{V}_{x} \mathrm{O}_{y}$ nanorods are formed with an average layer distance of about $0.7 \mathrm{~nm}$. The $\mathrm{V}_{x} \mathrm{O}_{y}$ nanorods exhibit a well-ordered crystalline structure. Nesper and co-workers reported the interlayer distances of vanadium oxide nanotubes prepared via hydrothermal treatment of the sol-gel method ranged from 1.7 to $3.8 \mathrm{~nm}$ and correlated linearly with the template size [8]. The template organic molecules are located in between the $\mathrm{VO}_{x}$ layers. Template-free $\mathrm{VO}_{x}$ nanotubes cannot be obtained by the classical thermal method of template removal because the structure of the assynthesized $\mathrm{VO}_{x}$ nanotubes collapses after calcination at $250{ }^{\circ} \mathrm{C}$ to an amorphous vanadium oxide even under protective atmosphere. The larger layer distance of $\mathrm{VO}_{x}$ nanotubes prepared by Nesper et al. than the as-synthesized $\mathrm{V}_{x} \mathrm{O}_{y}$ nanorods by $\mathrm{NH}_{4} \mathrm{VO}_{3}$ hydrolysis might be attributed 
to the insertion of organic template into the interlayer of vanadium oxide nanotubes. Fig. 5 shows the element mapping of a carbon nanotube and a bundle of nanorods. As seen in Fig. 5b, the nanotube consists of only carbon. However, the bundle of the nanorods is comprised of vanadium and oxygen (Fig. 5c and d). No vanadium is filled into the tubes or coated on the surface of the tubes.
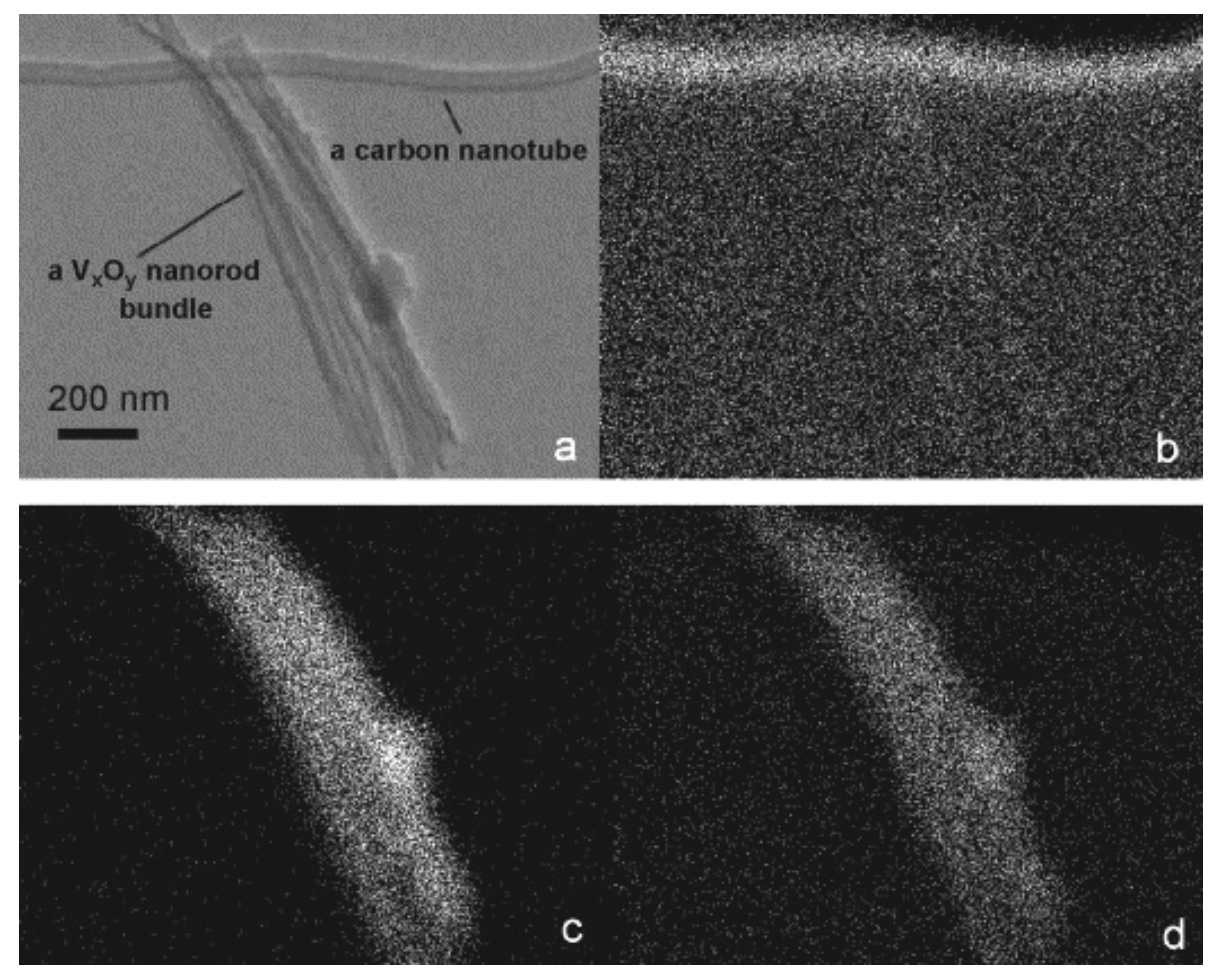

Fig. 5.(a) Scanning electron microscopy with energy dispersive X-ray analysis of vanadium oxide nanorods; (b) carbon; (c) vanadium and (d) oxygen.

Fig. 6(a) presents the PTR-MS response for the masses 71 and 99 amu during the step-wise heating of the product from $\mathrm{NH}_{4} \mathrm{VO}_{3}$ hydrolysis without carbon nanotubes in the reaction mixture of $n$-butane, oxygen and nitrogen. The signal corresponding to the protonated mass 99 amu can be unambiguously identified as maleic anhydride (molecule mass $98 \mathrm{amu}$ ). The signal at a protonated mass of $71 \mathrm{amu}$ (i.e. molecule mass $70 \mathrm{amu}$ ) corresponds to crotonaldehyde or 2,5-dihydrofuran. Crotonaldehyde is more likely because acetic acid was observed among the reaction products. It can be seen that the PTR-MS signals of 99 and 71 amu increase with increase of reaction temperature. The selectivity for the desired product, i.e. maleic anhydride versus the unwanted crotonaldelyde is about 1:3 on the product from $\mathrm{NH}_{4} \mathrm{VO}_{3}$ hydrolysis in absence of carbon nanotubes. Under similar reaction conditions, the nanostructured catalyst leads to higher ratio of expected product maleic anhydride to crotonaldelyde than with the nonnanostructured oxide. Heating to $400{ }^{\circ} \mathrm{C}$ apparently destroyed the vanadium oxide nanostructure and converted it into normal $\mathrm{V}_{2} \mathrm{O}_{5}$ (post-mortem analysis). The change in selectivity which is now poor with respect to maleic anhydride reveals that the reaction is sensitive to the microstructure of even the vanadium oxide. The final experiment of Fig. 6(b) at $300{ }^{\circ} \mathrm{C}$ illustrates clearly the extremely beneficial role of nanostructuring of vanadium oxide when used as oxidation catalyst. The data show that structurally complex variants of a vanadium oxide can be very attractive oxidation catalysts at lower temperatures than the non-nanostructured vanadium oxide obtained from $\mathrm{NH}_{4} \mathrm{VO}_{3}$ hydrolysis without carbon nanotubes. As mentioned above, the surface area of the hydrolysis product of $\mathrm{NH}_{4} \mathrm{VO}_{3}$ with CNTs is higher than that without CNTs. The higher selectivity and activity of the $\mathrm{V}_{x} \mathrm{O}_{y}$-CNT composite for $n$-butane to maleic anhydride are due to the nanostructured $\mathrm{V}_{x} \mathrm{O}_{y}$ and the higher surface area of the composite. The experiment also clearly demonstrates that the temperature required for a good 
catalytic performance depends neither on the organic substrate (not given by the thermodynamics of, e.g. the $\mathrm{C}-\mathrm{H}$ bonds) nor on the active element (vanadium in all cases) but to a very large extent on the correct structure of the catalyst which must be nanoscopic in dimension.
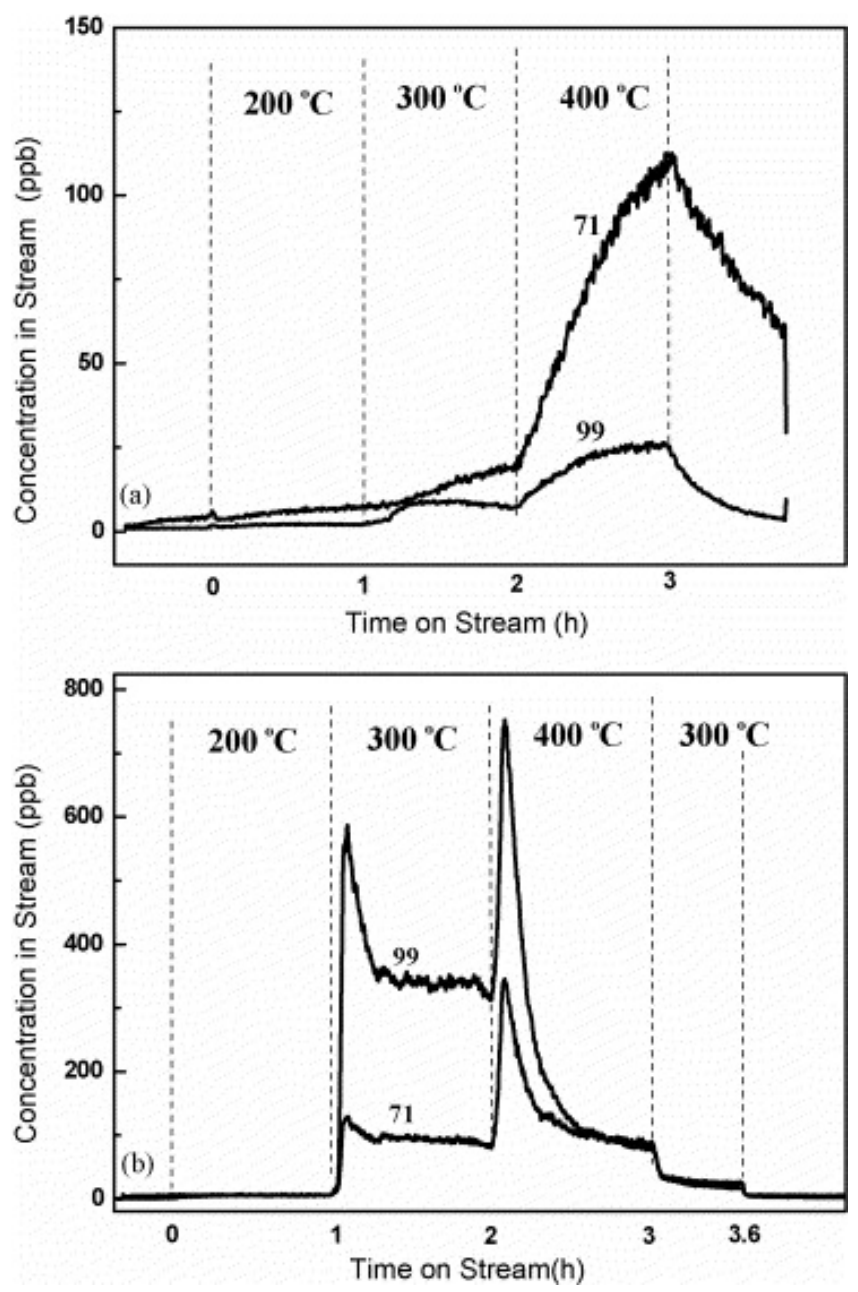

Fig. 6.Online PTR-MS response for masses 71 and 99amu while (a) product of $\mathrm{NH}_{4} \mathrm{VO}_{3}$ hydrolysis without carbon nanotubes and (b) the vanadium oxide nanorod-carbon nanotube composites were heated sequentially to 200, 300 and $400 \mathrm{C}$ in the reaction mixture of $n$-butane and oxygen.

The PTR-MS is not sensitive to the molecules $\mathrm{C}_{4} \mathrm{H}_{10}, \mathrm{O}_{2}, \mathrm{CO}$ and $\mathrm{CO}_{2}$, therefore the electron impact mass spectrometer is also used. However, decrease of $n$-butane signal was observed because of much lower sensitivity. A peak in the $\mathrm{CO}_{2}$ signal could be observed during the heating of vanadium oxide nanorod-carbon nanotube composite to $400^{\circ} \mathrm{C}$. This finding points to the combustion of carbon nanotubes. Obviously, this affects the active sites of the vanadium oxide nanorods as become apparent in a drastic decrease in activity and selectivity towards maleic anhydride (signal ratio 99/71 amu). The $\mathrm{V}_{x} \mathrm{O}_{y}$-CNT composites showed satisfactory level of stability during a 4-h test for partial oxidation of $n$-butane at $300{ }^{\circ} \mathrm{C}$. A slight change in concentration of maleic anhydride was observed and assigned to the defective morphology of CNTs.

\section{Conclusions}

Vanadium oxide nanorod-CNT composites were prepared by solution-based hydrolysis of $\mathrm{NH}_{4} \mathrm{VO}_{3}$ in the presence of CNTs. The CNTs exhibit catalytic behaviors and induce nucleation of $1 \mathrm{D}$ vanadium oxides, with the nuclei growing into long freestanding nanorods. The catalytic 
activity for $n$-butane partial oxidation of vanadium oxide nanorod-CNT composite was investigated. The result shows that the composite is active in partial oxidation of $n$-butane to maleic anhydride, and is more active at 300 than at $400{ }^{\circ} \mathrm{C}$, which is different from the nonnanostructured vanadium oxide sample obtained from $\mathrm{NH}_{4} \mathrm{VO}_{3}$ hydrolysis without carbon nanotubes. Although more quantitative evaluation of the catalytic performance is still needed, our first results may open a new horizon for the application of vanadium oxide-CNT composite as a potential catalyst in partial oxidation of $n$-butane.

\section{Acknowledgements}

This work is performed in frame of ELCASS and SFB 564 of DFG. Z.P. Zhu thanks Alexander von Humboldt Foundation for scholarship support. The authors thank Dr. M. Willinger for helpful discussion about EELS results.

\section{References}

[1] G. Mestl, N.I. Maksimova, N. Keller, V.V. Roddatis and R. Schlögl, Angew. Chem. Int. Ed. 40 (2001), p. 2066.

[2] M.E. Spahr, P. Bitterli, R. Nesper, M. Müller, F. Krumeich and H.U. Nissen, Angew. Chem. Int. Ed. 37 (1998), p. 1263.

[3] P.M. Ajayan, O. Stephan, P. Redlich and C. Colliex, Nature 375 (1995), p. 564.

[4] N. Pinna, M. Wilinger, K. Weiss, J. Urban and R. Schlögl, Nano Lett. 3 (2003), p. 1131.

[5] B.C. Satishkumar, A. Govindaraj, M. Nath and C.N.R. Rao, J. Mater. Chem. 10 (2000), p. 2115.

[6] F. Krumeich, H.-J. Muhr, M. Niederberger, F. Bieri, B. Schnyder and R. Nesper, J. Am. Chem. Soc. 121 (1999), p. 8324 .

[7] M.E. Spahr, P. Stoschitzki-Bitterli, R. Nesper, O. Haas and P. Vovák, J. Electrochem. Soc. 146 (1999), p. 2780.

[8] H.-J. Muhr, F. Krumeich, U.P. Schönholzer, F. Bieri, M. Niederberger, L.J. Gauckler and R. Nesper, Adv. Mater. $12(2000)$, p. 231.

[9] J.-F. Xu, R. Czerw, S. Webster, D.L. Carroll, J. Ballato and R. Nesper, Appl. Phys. Lett. 81 (2002), p. 1711.

[10] L.-Q. Mai, W. Chen, Q. Xu, J.-F. Peng and Q.-Y. Zhu, Chem. Phys. Lett. 382 (2003), p. 307.

[11] M.J. Ledoux, C. Crouzet, C. Pham-Huu, V. Turines, K. Kourtakis, P.L. Mills and J.J. Lerou, J. Catal. 203 (2001), p. 495.

[12] B. Solsona, T. Blasco, J.M. López Nieto, M.L. Peña, F. Rey and A. Vidal-Moya, J. Catal. 203 (2001), p. 443.

[13] B.M. Weckhuysen and D.E. Keller, Catal. Today 78 (2003), p. 25.

[14] T. Blasco and J.M. López Nieto, Appl. Catal. A: Gen. 157 (1997), p. 117.

[15] S. Jun, S.H. Joo, R. Ryoo, M. Kruk, M. Jaroniec, Z. Liu, T. Ohsuna and O. Terasaki, J. Am. Chem. Soc. 122 (2000), p. 10712.

[16] Z. Zhang, Y. Han, L. Zhu, R. Wang, Y. Yu, S. Qiu, D. Zhao and F.-S. Xiao, Angew. Chem. Int. Ed. 40 (2001), p. 1258.

[17] M. Kang, S.H. Yi, H.I. Lee, J.E. Yie and J.M. Kim, Chem. Commun. (2002), p. 1944.

[18] L. Wang, K. Lin, Y. Di, D. Zhang, C. Li, Q. Yang, C. Yin, Z. Sun, D. Jiang and F.-S. Xiao, Micropor. Mesopor. Mater. 86 (2005), p. 81. 
[19] H. Li, H. Xi, S. Zhu and R. Wang, Mater. Lett. 60 (2006), p. 943.

[20] X. Dong, W. Shen, J. Gu, L. Xiong, Y. Zhu, H. Li and J. Shi, Micropor. Mesopor. Mater. 91 (2006), p. 120.

[21] C. Minchev, H. Huwe, T. Tsoncheva, D. Paneva, M. Dimitrov, I. Mitov and M. Fröba, Micropor. Mesopor. Mater. 81 (2005), p. 333.

[22] Q. Hu, J. Pang, N. Jiang, J.E. Hampsey and Y. Lu, Micropor. Mesopor. Mater. 81 (2005), p. 149.

[23] J. Ding, K.-Y. Chan, J. Ren and F.-S. Xiao, Electrochim. Acta 50 (2005), p. 3131.

[24] J.S. Sakamoto and B. Dumn, J. Electrochem. Soc. 149 (2002), p. A26.

[25] W. Dong, J. Sakamoto and B. Dunn, J. Sol-gel Sci. Technol. 26 (2003), p. 641.

[26] G.W. Coulston, S.R. Bare, H. Kung, K. Birkeland, G.K. Bethke, R. Harlow, N. Herron and P.L. Lee, Science 275 (1997), p. 191.

[27] C.Y. Xiao, X. Chen, Z.Y. Wang, W.J. Ji, Y. Chen and C.T. Au, Catal. Today 93-95 (2004), p. 223.

[28] N. Govender, H.B. Friedrich and M.J. van Vuuren, Catal. Today 97 (2004), p. 315.

[29] C. Kuhrs, M. Swoboda and W. Weiss, Top. Catal. 15 (2001), p. 13.

[30] W. Lindinger, A. Hansel and A. Jordan, Öster. Phys. Ges. 2 (1998), p. 7.

[31] W. Lindinger, A. Hansel and A. Jordan, Int. J. Mass Spectrom. Ion Proc. 173 (1998), p. 191.

[32] J. Fink, Th. Müller-Heinzerling, B. Scheerer, W. Speier, F.U. Hillebrecht, J.C. Fuggle, J. Zaanen and G.A. Sawatzky, Phys. Rev. B 32 (1985), p. 4899. 